\title{
Resonant subgap current transport in Josephson field effect transistor
}

\author{
E. V. Bezuglyi, ${ }^{1,2}$ E. N. Bratus', ${ }^{1}$ and V. S. Shumeiko ${ }^{2}$ \\ ${ }^{1}$ B. Verkin Institute for Low Temperature Physics and Engineering, Kharkiv 61103, Ukraine \\ ${ }^{2}$ Chalmers University of Technology, S-41296 Göteborg, Sweden \\ (Received 5 December 2016; published 30 January 2017)
}

\begin{abstract}
We study theoretically the current-voltage characteristics (IVCs) of the Josephson field effect transistor-a ballistic SNINS junction with superconducting (S) electrodes confining a planar normal-metal region (N), which is controlled by the gate-induced potential barrier (I). Using the computation technique developed earlier for long single-channel junctions in the coherent multiple Andreev reflection (MAR) regime, we find a significant difference of the subgap current structure compared to the subharmonic gap structure in tunnel junctions and atomic-size point contacts. For long junctions, whose lengths significantly exceed the coherence length, the IVC exhibits current peaks at multiples (harmonics) of the distance $\delta_{m}$ between the static Andreev levels $e V_{n}=n \delta_{m}$. Moreover, the averaged IVC follows the powerlike behavior rather than the exponential one and has a universal scaling with the junction transparency. This result is qualitatively understood using an analytical approach based on the concept of resonant MAR trajectories. In shorter junctions having lengths comparable to the coherence length, the IVC has an exponential form common for point contacts, however the current structures appear at the subharmonics of the interlevel distance $e V_{n}=\delta_{m} / n$ rather than the gap subharmonics $2 \Delta / n$.
\end{abstract}

DOI: 10.1103/PhysRevB.95.014522

\section{INTRODUCTION}

In a number of experiments, the current-voltage characteristics (IVCs) were measured in superconductor-normal metal-superconductor (SNS) junctions consisting of a highmobility two-dimensional electron gas (2DEG) connected to two bulk superconducting electrodes [1-6]. In these devices, the electrodes have a large width $w$ up to $40 \mu \mathrm{m}$, whereas the distance $L$ between the electrodes varies between 0.2 and $1 \mu \mathrm{m}$. This is comparable or larger than the coherence length $\xi_{N}=\hbar v_{F} / \Delta$, where $v_{F}$ is the Fermi velocity in the 2DEG but much smaller than the elastic- and inelastic-scattering lengths. These junctions show well-pronounced Josephson effect and have rather small NS interface resistances which correspond to a large transmission coefficient of $\sim 0.8[4,6]$.

Most of these 2DEG structures were fabricated in effort to develop a Josephson field effect transistor (JOFET) [7]. In this device, schematically shown in Fig. 1(a) (see, e.g., Ref. [5]), the Josephson current is controlled by the gate voltage which changes the carrier concentration within the area just beneath the gate, thus, creating an effective potential barrier across the 2DEG layer. Such a device is a practical realization of the theoretical model of a wide superconductor-normal metalinsulator-normal metal-superconductor (SNINS) junction with a tunable potential barrier inside the 2D normal-metal layer sketched in Fig. 1(b), provided the barrier width is much smaller than $L$. The dc and ac Josephson currents in such junctions were studied in Refs. [8-10]. In our paper, we examine the dissipative charge transport through JOFET in the regime of a given applied voltage and predict a markedly different subgap current structure compared to point contacts and tunnel junctions, originating from Andreev bound states in the normal layer.

Assuming the dephasing length much longer than the junction length, we consider the coherent multiple Andreev reflection (MAR) regime, the theory of which has mostly been developed for the contacts with a single conducting channel: short junctions with the length $L \ll \xi_{N}$ [11-13] and long junctions with a strong scatterer [14]. In the multichannel transport regime in short junctions, the current can be evaluated by integrating the single-channel currents over the distribution of the normal junction transmission eigenvalues. The overall shape of the IVC depends on the transmission distribution: The qualitative result for the dc current in short contacts is exponential decay at a low voltage, $I \sim D^{2 \Delta / \mathrm{eV}}$, for various tunnel structures [11-13,15,16], whereas it is $I \sim V^{1 / 2}$ for diffusive constrictions [17]. Furthermore, the IVCs contain current structures at the gap subharmonics $e V=2 \Delta / n$ (subharmonic gap structure).

In long multichannel junctions one would expect that the current resonances found in the single-channel junction [14] will be averaged out after integration over channels, which is equivalent to averaging over their effective lengths. However, as we will show in this paper, the resonances survive due to the length cutoff of the conducting channels, whereas the averaging results in a universal scaling behavior of the IVC in the limit of long junctions, accompanied by essential enhancement of the subgap current.

The structure of the paper is the following. After a brief discussion of the MAR theory for a single channel and its generalization to the multichannel case in Sec. II, we present the results of the numerical calculation of the IVC of a multichannel junction in Sec. III. This calculation indicates the existence of two qualitatively different regimes. In a relatively short junction with only two Andreev levels for the majority of quasiparticles, the IVC has an exponential form and exhibits features at subharmonics of the maximum value $\delta_{m}$ of the interlevel distance. In the limit of a long junction (multilevel situation), the IVC is powerlike, and the resonant features occur at the voltages which are multiples (harmonics) of $\delta_{m}$. In order to explain the physics of these results, we develop in Sec. IV an analytical approach to the calculation of the IVC for long multichannel junctions. We show that the single- and two-particle currents scale as $R_{N}^{-1}$, where $R_{N} \sim D^{-1}$ is the normal resistance of the junction and the $n$-particle currents $(n>2)$ are described by 


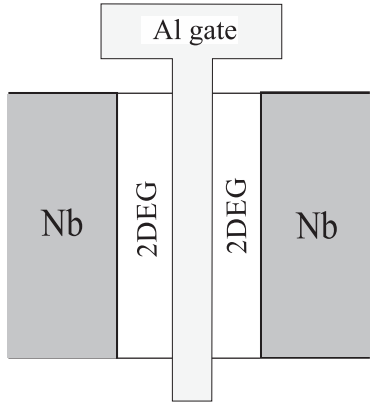

(a) (b)

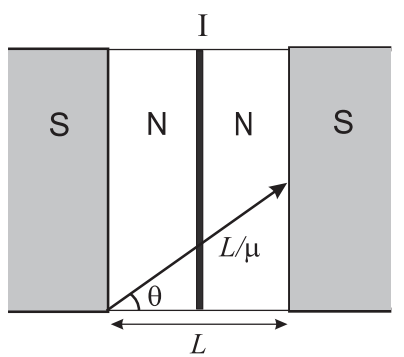

FIG. 1. (a) Experimental JOFET setup [5] and (b) the theoretical model of the gated SNS junction; the role of a controlled tunnel barrier (I) is played by the gate potential. The tilted arrow depicts the electron trajectory having length $L / \mu, \mu=\cos \theta$.

universal dependencies $I_{n}=\left(\Delta \sqrt{D} / e R_{N}\right) F_{n}(e V / \Delta)$, thus, scaling as $D^{3 / 2}$.

\section{CALCULATION SCHEME}

In this section, we briefly discuss our calculation method based on the results of calculation of the dc current for the voltage biased single-channel [one-dimensional (1D)] structure [14]. In the $1 \mathrm{D}$ case, the net current $I^{1 \mathrm{D}}(V)$ is expressed through the sum of the contributions of partial $n$-particle spectral currents $j_{n}(E)$,

$$
\begin{aligned}
I^{1 \mathrm{D}}= & -\frac{e}{h} \sum_{n=1}^{\infty} n\left[\Theta(n e V-2 \Delta) \int_{\Delta-n e V}^{-\Delta} d E j_{n}(E) \tanh \frac{E}{2 T}\right. \\
& \left.+\int_{-\infty}^{-\Delta-n e V} d E j_{n}(E)\left(\tanh \frac{E}{2 T}-\tanh \frac{E_{n}}{2 T}\right)\right],
\end{aligned}
$$

where $\Theta(x)$ is the Heaviside step function and

$$
\begin{aligned}
j_{n}(E) & =\frac{8 \xi \xi_{n}}{\Delta^{2}|Z(E)|^{2}}\left(e^{\gamma}+\left|r_{0-}\right|^{2} e^{-\gamma}\right)\left(e^{\gamma_{n}}+\left|r_{n+}\right|^{2} e^{-\gamma_{n}}\right), \\
Z(E) & =\left(1,-r_{0-}\right)\left(\hat{U}_{n} \hat{M}_{n 0} \hat{U}_{0}\right)^{-1}\left(\begin{array}{c}
1 \\
r_{n+}
\end{array}\right) .
\end{aligned}
$$

Here index $n$ indicates the shift of the energy $E_{n}=E+$ $n e V, \exp (\gamma)=(E+\xi) / \Delta$, and $\xi(E)=\sqrt{(E+i 0)^{2}-\Delta^{2}}$ is the analytical function of the complex-valued energy defined in the upper half-space. The amplitudes $r_{n \pm}$ refer to the limiting values of the following ratios of matrix elements of the $2 \times 2$ matrix $\hat{M}_{n m}$ :

$$
r_{n+}=-\lim _{m \rightarrow+\infty} \frac{\left(\hat{M}_{m n}\right)_{11}}{\left(\hat{M}_{m n}\right)_{12}}, \quad r_{0-}=\lim _{m \rightarrow-\infty} \frac{\left(\hat{M}_{0 m}\right)_{12}}{\left(\hat{M}_{0 m}\right)_{22}} .
$$

In practical calculations, the limits in Eq. (3) are truncated according to the rule: The energies $E_{m}$ and $E_{n}$ defined by the indices of the matrix $\hat{M}_{m n}$ are to be located at different sides of the energy gap with few added steps in $m$ for better accuracy.

The matrix $\hat{M}_{n m}(\operatorname{det} \hat{M}=1, n>m)$ has the meaning of a transfer matrix along the MAR trajectory in the energy space across the gap [18] and is defined as the product,

$$
\begin{gathered}
\hat{M}_{n m}=\hat{T}_{n-1} \hat{U}_{n-1} \hat{T}_{n-2} \hat{U}_{n-2} \cdots \hat{T}_{m}, \\
\hat{U}(E)=e^{-\sigma_{z} \gamma}, \quad \hat{T}_{2 n}=\left(\hat{T}_{2 n}^{e}\right)^{-1}, \quad \hat{T}_{2 n+1}=\hat{T}_{2 n+1}^{h} .
\end{gathered}
$$

Here the matrices $\hat{U}_{n}(E)=\hat{U}\left(E_{n}\right)$ describe the phase shifts of the wave functions associated with the Andreev reflection, and $\hat{T}_{n}^{e, h}=\hat{T}^{e, h}\left(E_{n}\right)$ are the real-space transfer matrices of the junction in the normal state for electrons and holes, respectively. We will consider a purely ballistic limit, neglecting electron scattering on random impurities; the potential barrier is modeled by a linear scatterer localized at the middle of the normal region and characterized with the energy-independent scattering amplitudes, which will be assumed identical for all conducting channels. Furthermore, we consider transparent NS interfaces, which eliminates the Fabry-Pérot interference effects and enables us to exclude the scattering phases, thus, reducing the characteristics of the scatterer to its transparency $D$ and reflection coefficient $R=1-D$. Accordingly, the transfer-matrices $\hat{T}$ in Eq. (5) are composed with the transfermatrix $\hat{t}$ of the scatterer and the ballistic transfer-matrices $\hat{u}(E)$, which describe free propagation through the normal region,

$$
\begin{aligned}
\hat{T}^{e, h}(E) & =\hat{u}^{e, h}(E) \hat{t} \hat{u}^{e, h}(E), \quad \hat{t}=D^{-1 / 2}\left(1+\sigma_{x} \sqrt{R}\right), \\
\hat{u}^{e, h}(E) & =\exp \left[i\left(p_{x} L \mp \sigma_{z} \frac{\varphi}{2}\right)\right], \\
\varphi(E) & =\frac{L}{v_{x}}\left(E+\frac{e V}{2}\right) .
\end{aligned}
$$

In planar junctions, $v_{x}$ and $p_{x}$ refer to the longitudinal (perpendicular to the barrier) components of the particle velocity and momentum, respectively. Then the matrix $\hat{T}_{n}$ can be written as

$$
\hat{T}_{n}=D^{-1 / 2}\left[\exp \left(i \sigma_{z} \varphi_{n}\right)-(-1)^{n} \sigma_{x} \sqrt{R}\right] .
$$

One can rewrite Eqs. (5)-(7) in a more convenient form by combining the matrices $\hat{u}^{e, h}$ with the $\hat{U}$ matrices rather than the $\hat{t}$ matrices. In such a representation,

$$
\begin{aligned}
\hat{T}_{n} & =D^{-1 / 2}\left[1-(-1)^{n} \sigma_{x} \sqrt{R}\right], \\
\hat{U}_{n} & =e^{-\sigma_{z}\left(\gamma_{n}-i E_{n} L / v_{x}\right)} .
\end{aligned}
$$

Along with this transformation, we exclude the factors with unity moduli from $Z(E)$ in Eq. (2).

Proceeding with the summation over the channels, we assume their number $\mathcal{N}$ to be macroscopically large. Enumerating the channels by the values of their transversal momentum $p_{\perp}$, which is quantized in a multichannel $2 \mathrm{D}$ junction of width $w$ as $p_{\perp k}=\pi k \hbar / w, k=0,1, \ldots$, we arrive at the following rule of the summation over the channels in the quasiclassical limit $\mathcal{N}=w p_{F} / \pi \hbar \gg 1$ where $p_{F}$ is the Fermi momentum:

$$
\begin{aligned}
I^{2 \mathrm{D}} & =\sum_{p_{\perp k}<p_{F}} I^{1 \mathrm{D}}\left(v_{x, k}\right) \rightarrow \frac{w}{\pi \hbar} \int_{0}^{p_{F}} d p_{\perp} I^{1 \mathrm{D}}\left(v_{x}\right) \\
& =\mathcal{N} \int_{0}^{1} d \mu q(\mu) I^{1 \mathrm{D}}\left(v_{F} \mu\right), \quad q(\mu)=\frac{\mu}{\sqrt{1-\mu^{2}}} .
\end{aligned}
$$

Here $\mu$ is the cosine of the angle $\theta$ of incidence of the quasiparticle at the NS boundary as shown in Fig. 1. For a 
three-dimensional (3D) structure with the cross-sectional area $S$ and $\mathcal{N}=\pi S p_{F}^{2} /(2 \pi \hbar)^{2}$,

$$
\begin{aligned}
I^{3 \mathrm{D}} & =\sum_{p_{\perp k l}<p_{F}} I^{1 \mathrm{D}}\left(v_{x, k l}\right) \\
& \rightarrow \frac{\pi S}{2(\pi \hbar)^{2}} \int_{0}^{p_{F}} p_{\perp} d p_{\perp} I^{1 \mathrm{D}}\left(v_{x}\right) \\
& =\mathcal{N} \int_{0}^{1} d \mu q(\mu) I^{1 \mathrm{D}}\left(v_{F} \mu\right), \quad q(\mu)=2 \mu .
\end{aligned}
$$

The quantity $\mathcal{N}$ can then be excluded through the relation to the normal conductance $R_{N}^{-1}=\left(2 e^{2} / h\right) D \mathcal{N}$, which is a sum of contributions $\left(2 e^{2} / h\right) D$ of separate conducting channels in the normal state.

In what follows, we assume the temperature to be much smaller than the energy gap $2 \Delta$ in the superconducting electrodes, which results in the following expression used in our calculations of the dc current:

$$
\begin{aligned}
I & =\frac{1}{e R_{N}} \sum_{n \geqslant 2 \Delta / e V} \int_{0}^{1} d \mu q(\mu) J_{n}, \\
J_{n} & =\frac{n}{D} \int_{-n e V / 2}^{-\Delta} d E j_{n}(E) .
\end{aligned}
$$

In Eq. (11) we used the symmetry of the spectral density $j_{n}(E)$ in Eq. (2) with respect to the middle of the initial integration interval $\Delta-n e V<E<-\Delta$.

\section{NUMERICAL RESULTS}

In Fig. 2 we present the calculated IVCs for three values of the transmission coefficients and lengths of the planar (2D) SNINS junction (the results for 3D junctions are similar). For a short junction $L=0.3 \xi_{N}$, the current rapidly decays with decreasing voltage, similar to the case of a short single-channel contact; in the limit $L \rightarrow 0$, the results for all dimensions coincide (being normalized by $R_{N}$ ). On the contrary, for comparatively long junctions $L=4 \xi_{N}$ and $L=10 \xi_{N}$, the current decreases with voltage much more slowly. Another interesting observation is that in the limit $L \gg \xi_{N}$, the smooth part of the IVC becomes independent of the junction length.

The most of characteristic features of the IVCs in the junction of finite length and width can be explained by the existence of discrete Andreev levels in the equilibrium junction and corresponding singularities in the density of the quasiparticle states. It is instructive to qualitatively examine highly resistive junctions where this connection is most prominent. In such junctions, the Andreev level spectrum can be approximated with the de Gennes-Saint-James levels [19] in each half of the normal layer with the length $L / 2$ (the level splitting due to finite barrier transparency will be discussed later). The dispersion equation for these levels coincides with the one for a SNS junction of equivalent length $L$,

$$
\frac{E L}{\Delta \mu \xi_{N}}=\pi n+\arccos \frac{E}{\Delta}, \quad n=0, \pm 1, \pm 2, \ldots,
$$

where $|E|<\Delta, \mu=\cos \theta$ and $\theta$ is the angle between the particle velocity and the normal to the junction interfaces.

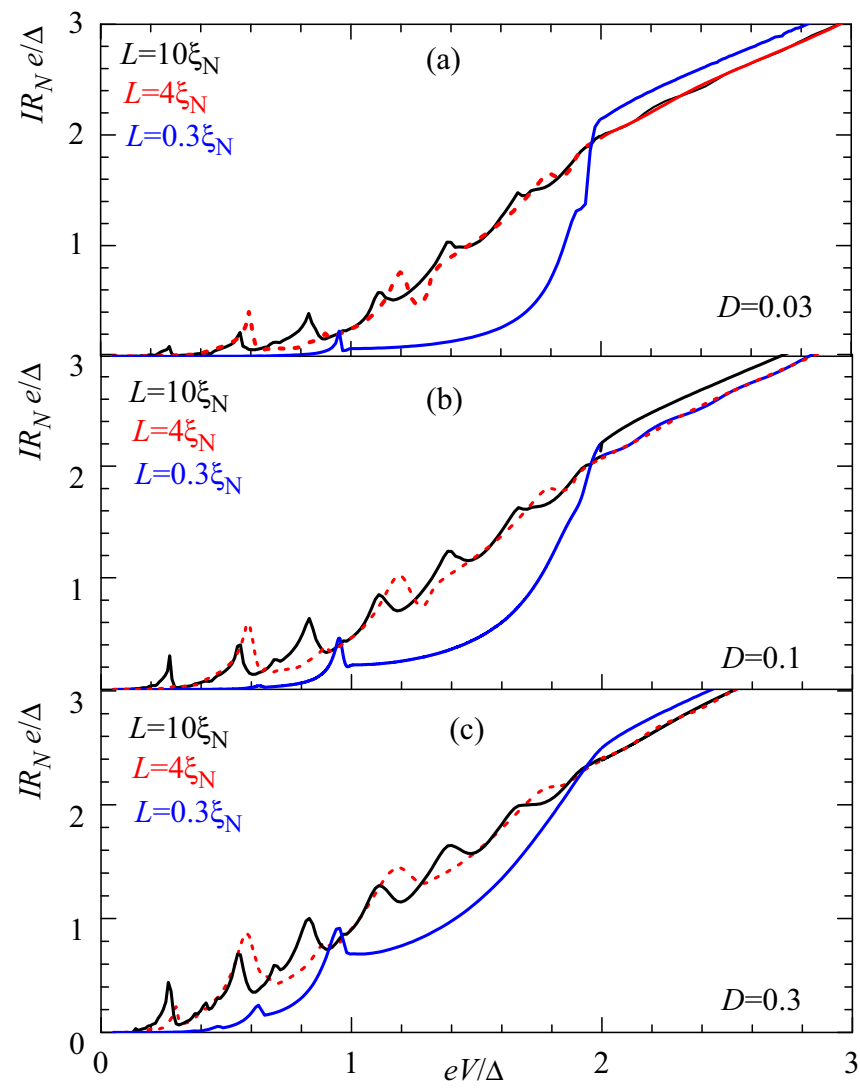

FIG. 2. Current-voltage characteristics for different transmission coefficients (a) $D=0.03$, (b) 0.1 , (c) 0.3 , and lengths of the junction: $L / \xi_{N}=0.3$ (lower solid blue curves), 4 (red dashed curves), and 10 (upper solid black curves).

From Eq. (12) we obtain simple estimates for the average interlevel distance $\delta$ and the number of Andreev levels $n_{A}$,

$$
\begin{gathered}
\delta(L, \mu) \approx \frac{\Delta}{L / \pi \mu \xi_{N}+1 / 2}, \\
n_{A}(L, \mu)=2\left[\operatorname{Int}\left(L / \pi \mu \xi_{N}\right)+1\right],
\end{gathered}
$$

$[\operatorname{Int}(x)$ denotes the integer part of $x]$. The evolution of Andreev levels with the increase in the trajectory length $L / \mu$ is depicted in Fig. 3.

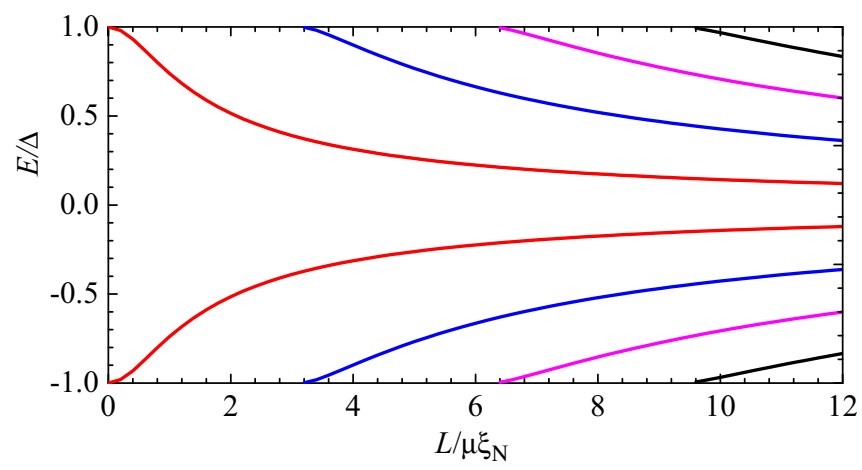

FIG. 3. Emergence and evolution of Andreev levels with the increase in the quasiparticle trajectory length. 


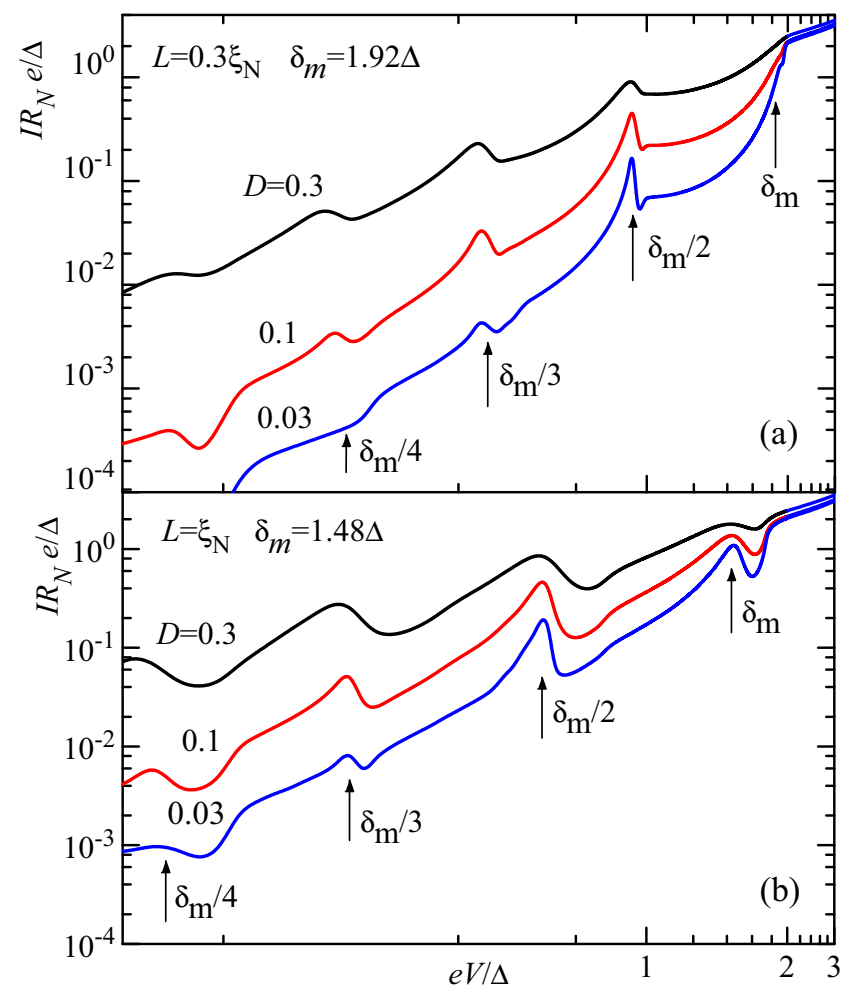

FIG. 4. Current structures corresponding to subharmonics $e V_{n}=\delta_{m} / n$ of the maximum interlevel distance $\delta_{m}$ for short junctions with the lengths (a) $L=0.3 \xi_{N}$ and (b) $L=\xi_{N}$ at different transmission coefficients.

As follows from Fig. 4, the features on the IVC of relatively short junctions correspond to the subharmonics $e V=\delta_{m} / n, n=1,2, \ldots$ of the maximum distance between two Andreev levels present in the shortest electron trajectory $\delta_{m}=\delta(L, 1)<2 \Delta$, which is quantitatively different from the common structure of the energy-gap subharmonics. Obviously, these features are due to the resonant MAR trajectories in the energy space whose first and last Andreev reflections occur in the neighborhood of the Andreev levels where the density of states is singular. A similar effect has been found in the diffusive SNcNS junction [20] where the role of the potential barrier was played by the ballistic constriction (c), and the role of Andreev levels_-by the edges of the proximity-induced minigap in diffusive normal-metal banks with greatly enhanced density of states; the appearance of the minigap subharmonics has also been predicted for a short diffusive double-barrier junction [21]. Furthermore, the averaged subgap IVC can be well approximated with the exponential dependence $I(V) \sim D^{\text {const } / e V}$, similar to the case of $L=0$ but with the constant close to $\delta_{m}$ rather than to $2 \Delta$.

Quite another picture of the IVC shape was found in longer junctions where the number of Andreev levels is comparatively large: $n_{A}=4$ for $L=4 \xi_{N}$, and $n_{A}=8$ for $L=10 \xi_{N}$. In this case, the IVCs plotted in Fig. 5 are nonexponential, and the resonant features correspond to multiples of the maximum interlevel distance $e V=n \delta_{m}, n=1,2, \ldots$, or, in other words, to harmonics of $\delta_{m}$. From this we conclude that the enhanced multiparticle charge transfer is entirely

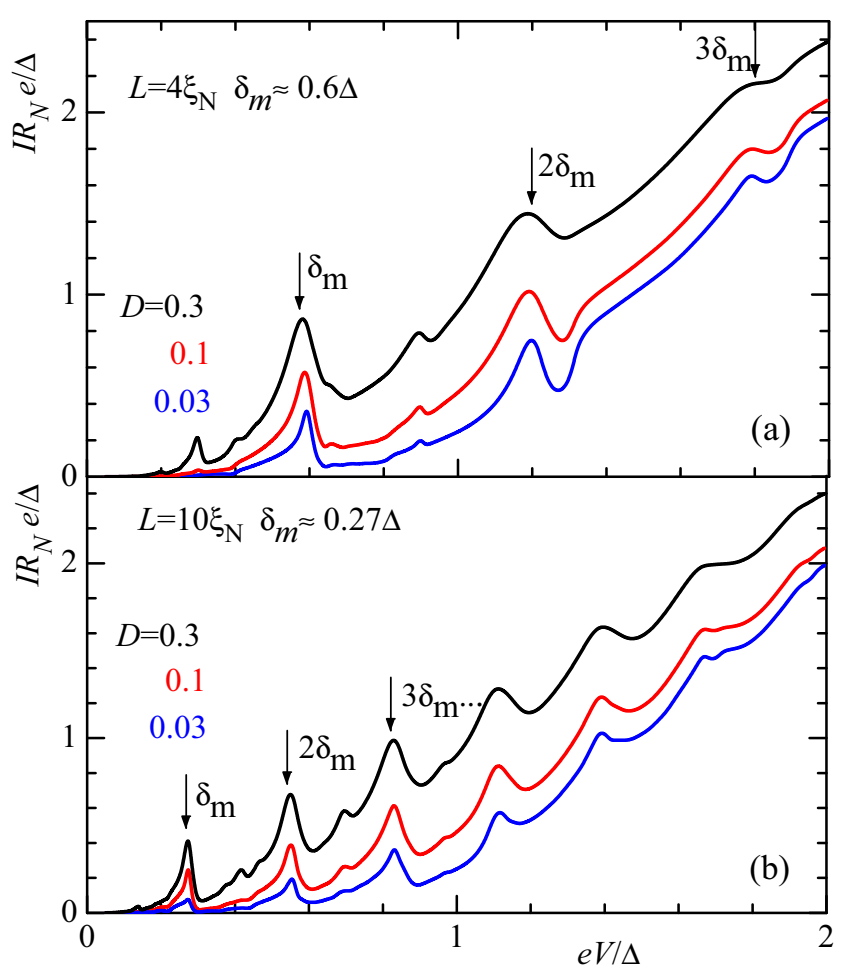

FIG. 5. Subgap features at multiples of the maximum interlevel distance $\delta_{m}$ in the IVCs of long junctions with the lengths (a) $L=4 \xi_{N}$ and (b) $L=10 \xi_{N}$ at different transmission coefficients.

due to the resonant MAR trajectories for which all points of Andreev reflections in the energy space coincide with the level positions. We note that at very low voltages or transparencies, the nonequidistance of the Andreev levels plays a role, and the fully resonant trajectories do not exist (for a more detailed analysis, see Ref. [14]). This leads to a rapid decrease in the subgap current at low voltages clearly visible in Fig. 5 at $e V \lesssim 0.3 \Delta$.

The next intriguing feature of the IVCs for long junctions is their approximate overlap at $e V<\Delta$ for different $D$, provided $I(V)$ is normalized over $\sqrt{D} / R_{N}$ as shown in Fig. 6. Note that the smaller $D$ is, the better this "universality" looks. This fact as well as the universality of the averaged IVCs for large $L$ mentioned above motivated us to develop the analytical theory which gives a clear physical explanation of the numerically found peculiarities in the IVCs.

\section{ANALYTICAL RESULTS}

\section{A. IVC at $e V \gg \Delta$ : excess current}

We start with evaluation of the excess current, i.e., voltageindependent deviation of the total current from the ohmic IVC at high applied voltage $e V \gg \Delta$. At these voltages, an analytical result in the finite form can be obtained for all lengths and transparencies because only the first two partial currents $J_{1}$ and $J_{2}$ give nonvanishing contributions to the net current and the amplitudes $r_{n \pm}$ approach the asymptotic values $r_{0-}=\sqrt{R}, r_{n+}=(-1)^{n} \sqrt{R}$. Within the first approximation, the current $J_{1}$ is given by $e V$, which results in a normal Ohm's 


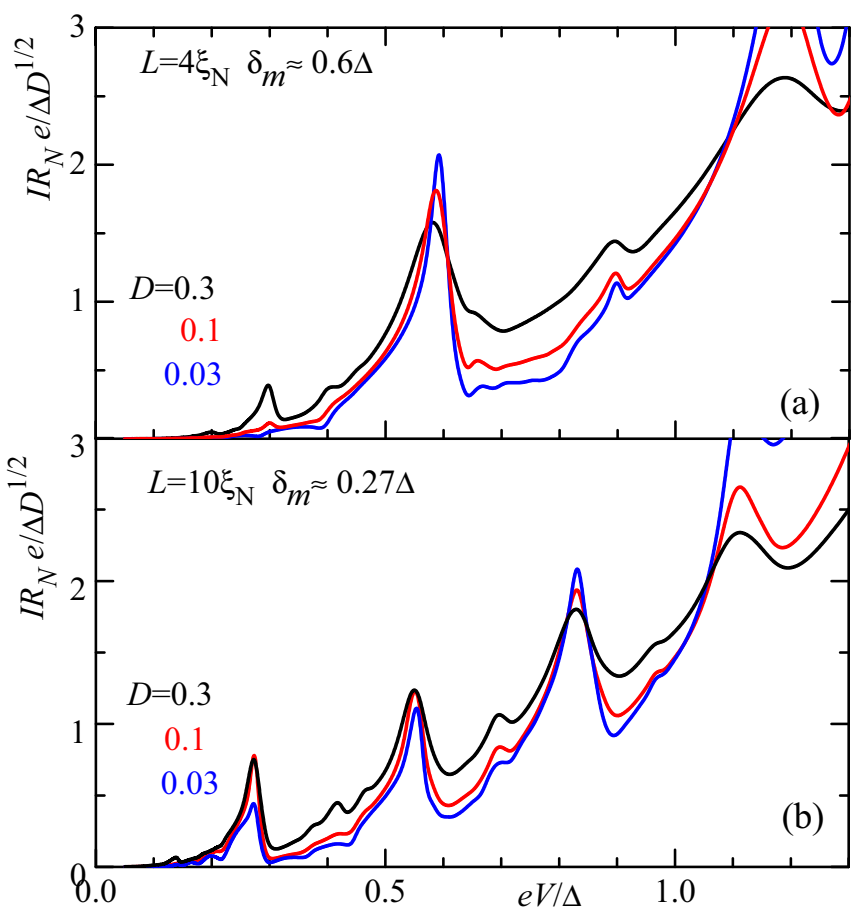

FIG. 6. Universality of the IVCs normalized over $\sqrt{D} / R_{N}$ at $e V<\Delta$ for the junction lengths (a) $L=4 \xi_{N}$ and (b) $L=10 \xi_{N}$ at different transmission coefficients.

law for the net current $I$. The contribution of $J_{1}$ to the excess current is related to the next term in its expansion over $\Delta / \mathrm{eV}$ associated with the energies $E \sim \Delta$,

$$
\begin{aligned}
& J_{1}^{\text {exc }} \\
& \quad=2 \int_{\Delta}^{\infty} d E\left\{\frac{2 \xi[2 E-D(E-\xi)]}{[2 \xi+D(E-\xi)]^{2}+4 R \Delta^{2} \sin ^{2} \phi}-\frac{E}{\xi}\right\}, \\
& \phi(E, \mu)=E L / \Delta \xi_{N} \mu .
\end{aligned}
$$

The contribution of the two-particle current consists of two parts in which the energy changes inside and outside the gap, respectively. The last contribution is combined with $J_{1}^{\text {exc }}$, and the general formula for the excess current reads

$$
\begin{aligned}
I^{\mathrm{exc}} & \frac{2}{e R_{N}} \int_{0}^{1} d \mu q(\mu)\left(2 D \int_{0}^{\Delta} \frac{d E}{D^{2}+4 R \sin ^{2}[\gamma(E)-\phi]}\right. \\
& \left.+\int_{\Delta}^{\infty} d E\left\{\frac{2 E[2 \xi+D(E-\xi)]}{[2 \xi+D(E-\xi)]^{2}+4 R \Delta^{2} \sin ^{2} \phi}-\frac{E}{\xi}\right\}\right),
\end{aligned}
$$

where $\gamma(E)=\arccos (E / \Delta)$. The behavior of the excess current as a function of the junction length is shown in Fig. 7.

In the limit of a short junction $L \ll \xi_{N}$, the main approximation in Eq. (16) reproduces known result for a single-mode junction (in terms of the normal junction resistance),

$$
I^{\mathrm{exc}}=\frac{\Delta}{e R_{N}} \frac{D}{R}\left[1-\frac{D^{2}}{2 \sqrt{R}(1+R)} \ln \frac{1+\sqrt{R}}{1-\sqrt{R}}\right], \quad L \ll \xi_{N} .
$$

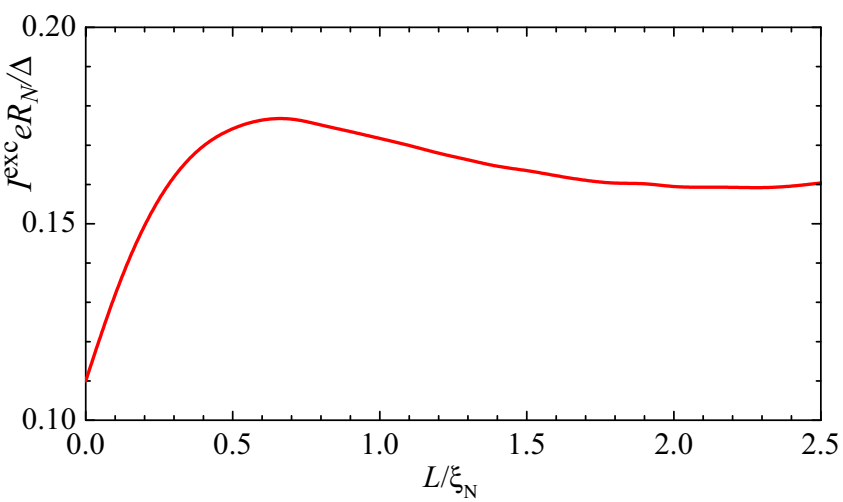

FIG. 7. Excess current vs junction length for the transparency $D=0.1$. The values of $I^{\text {exc }}$ in the limits $L \ll \xi_{N}$ and $L \gg \xi_{N}$ are 0.11 and 0.16, respectively, in agreement with Eqs. (17) and (23).

$$
I^{\mathrm{exc}} \approx \frac{\Delta}{e R_{N}} \begin{cases}D, & D \ll 1, \\ 8 / 3, & R \ll 1 .\end{cases}
$$

In the case of a long junction $L \gg \xi_{N}$, the integrands in Eq. (16) involve rapidly oscillating functions of $E$ and $\mu$. In order to obtain an analytical result within the main approximation in $\xi_{N} / L$, we use the following approach. Let us consider the integral,

$$
\begin{aligned}
A(\lambda) & =\int_{a}^{b} d x f[x, \lambda z(x)], \quad \lambda \gg 1, \\
f(x, y+2 \pi) & =f(x, y),
\end{aligned}
$$

where $f(x, y)$ and $z(x)$ smoothly vary at the distances much larger than $\lambda^{-1}$. First, we introduce $z$ as a new variable and split the whole interval of $z$ over small intervals of the length $p=2 \pi / \lambda \ll 1$,

$$
\begin{aligned}
A(\lambda)= & \sum_{k=0}^{k_{\max }-1} \int_{z(a)+k p}^{z(a)+(k+1) p} d z f[x(z), \lambda z] \frac{d x(z)}{d z} \\
& +\int_{z(a)+k_{\max } p}^{z(b)} d z f[x(z), \lambda z] \frac{d x(z)}{d z}, \\
& k_{\max } p<z(b)-z(a) .
\end{aligned}
$$

The second integral is of the order of $p \ll 1$ and can be neglected. In the first term, we shift $z \rightarrow z+k p$ and use the periodicity of $f(x, y)$ with respect to $y$. Then, due to smoothness of the functions $f(x, y)$ and $z(x)$ with respect to $x$, we approximate the summation over $k$ by the integration over $z$ and then return to the integration over $x$ within the initial interval $(a, b)$, whereas the integral over the second variable reduces to averaging over the phase $u=\lambda z$,

$$
A(\lambda) \approx \int_{a}^{b} d x \int_{0}^{2 \pi} \frac{d u}{2 \pi} f(x, u) .
$$

Within such an approximation, the first term in Eq. (16) is

$$
2 D \int_{0}^{\Delta} d E \int_{0}^{2 \pi} \frac{d u}{2 \pi} \frac{1}{D^{2}+4 R \sin ^{2}[\gamma(E)-u]}=\frac{2 \Delta}{1+R},
$$


whereas the second one is

$$
\begin{aligned}
& \int_{\Delta}^{\infty} d E \int_{0}^{2 \pi} \frac{d u}{2 \pi}\left\{\frac{2 E[2 \xi+D(E-\xi)]}{[2 \xi+D(E-\xi)]^{2}+4 R \Delta^{2} \sin ^{2} u}-\frac{E}{\xi}\right\} \\
& =-\Delta \frac{1+R}{2 R}\left(1-\frac{D}{\sqrt{R}} \arctan \sqrt{R}\right),
\end{aligned}
$$

which yields the final expression for the excess current,

$$
I^{\mathrm{exc}}=\frac{\Delta}{e R_{N}} \frac{D}{R}\left(\frac{1+R}{\sqrt{R}} \arctan \sqrt{R}-\frac{D}{1+R}\right), \quad L \gg \xi_{N},
$$

$$
I^{\mathrm{exc}} \approx \frac{\Delta}{e R_{N}} \begin{cases}\pi D / 2, & D \ll 1, \\ 8 / 3, & R \ll 1 .\end{cases}
$$

By contrast to the case $L \ll \xi_{N}$ where both contributions to the excess current are small $(\sim D)$ at small transparencies $D$, the main terms in Eqs. (21) and (22) are on the order of unity. However, they have opposite signs and almost compensate each other, which results in a small value $\left(\sim D^{2}\right)$ of the excess current in both limits. We note also that the expressions for $I^{\text {exc }}$ coincide for the short and long junctions in the limit of high transparency $R \ll 1$.

\section{B. Resonance approximation}

As we have seen from the numerical results, the subgap IVC of the short multichannel junction $L \lesssim \xi_{N}$ is qualitatively similar to the IVC of a single-channel point contact with the maximum interlevel distance $\delta_{m}$ replacing the superconducting energy-gap $2 \Delta$ and appropriately defined normal resistance $R_{N}$. A more interesting case, which allows us to obtain analytical results for arbitrary voltages, is the limit of a long junction $L \gg \xi_{N}$ when the subgap charge transfer is enhanced due to the existence of the resonant MAR trajectories which cross the energy gap through the chain of Andreev levels.

The single-particle current $I_{1}$ represents an exclusion: It does not involve Andreev levels and therefore always has a nonresonant nature. At $D \ll 1$ we have

$$
I_{1}=\frac{2}{e R_{N}} \Theta(e V-2 \Delta) \int_{0}^{1} d \mu q(\mu) \int_{-e V / 2}^{-\Delta} d E N N_{1},
$$

where the function,

$$
N(E, \phi)=\frac{E \xi}{\xi^{2}+\Delta^{2} \sin ^{2} \phi(E)}
$$

has the meaning of the local density of states. At $L \gg \xi_{N}$, we apply the phase averaging method described in the previous section to perform integration over the energy and angle. This is performed by averaging the integrand over two independent phases $\phi_{0}$ and $\phi_{1}$. This gives for $I_{1}(V)$ a linear ohmic dependence starting from the threshold point $e V=2 \Delta$,

$$
I_{1}=\frac{e V-2 \Delta}{e R_{N}} \Theta(e V-2 \Delta) .
$$

Proceeding with multiparticle currents $I_{n}, n>1$ and taking into account only fully resonant trajectories, we must require the energies $E_{k}$ of all intermediate Andreev reflections $(1 \leqslant k \leqslant n-1)$ to be located inside the gap where the Andreev levels exist (actually, this means elimination of the MAR trajectories with partial over-the-barrier Andreev reflections above the energy gap). This results in the restriction $E<E_{\max }$ for the initial energy of the MAR chain and in a limited voltage range where the $n$-particle current is nonzero,

$$
\begin{aligned}
I_{n} & =\frac{n D^{n-1}}{e R_{N}} \int_{-n e V / 2}^{E_{\max }} d E\left\langle\frac{8 N N_{n}}{|Z|^{2}}\right\rangle, \\
E_{\max } & =\min [-\Delta, \Delta-(n-1) e V], \\
2 \Delta / n & \leqslant e V \leqslant 2 \Delta /(n-2),
\end{aligned}
$$

where the angle brackets denote averaging over the two phases mentioned above.

The two-particle current is a special one and differs from the high-order currents: It involves only one intermediate Andreev reflection, and therefore only the initial energy $E$ should be adjusted in order to achieve the resonant transmission when the energy $E_{1}=E+e V$ coincides with the Andreev level; the interlevel distance is irrelevant in this aspect. Formally, this manifests itself in the appearance of only one resonant phase in the denominator; a characteristic scale for this phase is $D$. The second independent phase is nonresonant and varies within the whole interval of periodicity,

$$
\begin{aligned}
I_{2} & =\frac{4}{e R_{N}} \Theta(e V-\Delta) \int_{0}^{2 \pi} \frac{d u}{2 \pi} \int_{0}^{\min (\Delta, e V-\Delta)} \frac{d E}{N_{+}^{-1}+N_{-}^{-1}}, \\
N_{ \pm} & =N\left(E \pm e V, \phi_{ \pm}\right), \quad \phi_{ \pm}=\gamma(E) \pm u
\end{aligned}
$$

[for symmetry, we used $E_{1}$ as the integration variable $E$ in Eq. (29)]. We note that, due to resonant transmission through the intermediate Andreev level, the two-particle current appears to be of similar order $D$ as the single-particle one. This result was discovered already during the calculation of the excess current; the latter, however, was found to be on the order of $D^{2}$ due to the cancellation of the main terms in the two-particle current and in the "deficit" part of the one-particle current.

For high-order partial currents $n>2$, both the energy of quasiparticles and the interlevel distance (the propagation angle) should satisfy the resonant conditions for a given voltage, therefore two independent phases (other phases contain their linear combinations) are always resonant. Several results obtained with such an approach are presented in the Appendix.

\section{IVC at $e V<\Delta$}

The formal phase averaging method described above enables us to reproduce only the smooth part of the IVC; furthermore, the calculation of $I_{n}$ requires a separate approach for each $n$, and the complexity of expressions for $I_{n}$ enormously increases with $n$. In this section, we formulate another semiquantitative approach to the description of the IVC at $e V<\Delta$, which gives a more detailed and clear physical explanation of the resonant charge transmission. Within this approach we obtain simple and universal formulas showing good agreement with the results of numerical calculations, including the resonant features of the IVC. This approach is based on the analysis for the single-channel contact [14], which shows that the IVC exhibits a complex pattern of current peaks resulting from the multiple Andreev resonances. Integration over the angle will smoothen this pattern; however, one should 


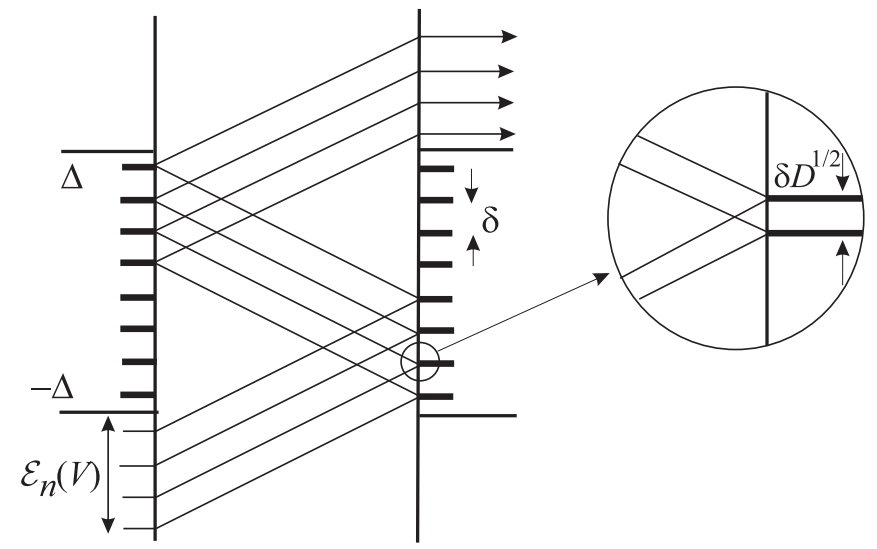

FIG. 8. Resonant MAR trajectories passing through the Andreev levels (short bold lines) and contributing to the three-particle current at $L=10 \xi_{N}$ and $e V=4 \delta$. The inset shows splitting of a static Andreev level to the cluster consisting of two levels, therefore the whole number of the resonant trajectories is 8 in this case.

expect that the reduced oscillations will remain since the geometric weight $q(\mu)$ of the partial conducting channels has the threshold at the reference point $\theta=0(\mu=1)$. We will perform our estimations and analytical calculations within the model of the equidistant Andreev spectrum $\delta(E)=$ const, which formally corresponds to approximating of $\arccos (E / \Delta)$ in Eq. (12) with a linear function. This model is justified by the numerical fact that, for all transparencies (except for very small ones), the energy width of the multiparticle resonances is comparable with the nonequidistance of the Andreev levels.

As before, we will consider the resonant MAR paths when all $n-1$ Andreev reflections occur at the resonance energies within the superconducting gap, i.e., the voltage is assumed to be commensurate with the level spacing $e V=k \delta$, and it belongs to the interval $V_{n}<V<V_{n-2}\left(V_{n}=2 \Delta / n\right)$. Such trajectories, shown in Fig. 8 for the three-particle current, give the main contribution to the current spectral density $j_{n} \sim D(E, E+n e V)$ where the quantity,

$$
D\left(E_{m}, E_{n}\right)=\left|\left(\hat{M}_{m n}\right)_{11}\right|^{-2}
$$

has the meaning of the effective transparency of MAR chains between the points $E_{n}$ and $E_{m}>E_{n}$ at the energy axis [14]. This resonant MAR process is mapped onto a periodic $n$ barrier tunnel structure with resonant levels in each well having the same energy when the barriers are nontransparent [18]. At finite barrier transparency, the levels will repel each other forming a tight cluster of resonant levels within the energy interval $\sim \delta \sqrt{D}$ (precursor of the energy band at $n \rightarrow \infty$ ) as shown in the inset in Fig. 8. This leads to the splitting of the resonant transmissivity of the whole structure into $n-1$ peaks at the energy axis and results in the following schematic structure of the function:

$$
D\left(E, E_{n}\right)=\frac{D^{n}}{\prod_{k=1}^{n-1}\left(\varepsilon / \delta-a_{k} \sqrt{D}\right)^{2}+\Lambda^{2}}
$$

in the vicinity of the cluster for a single conducting channel [14]. Here $a_{k}$ are numerical constants describing distribution of the resonant levels over the cluster, and $\varepsilon$ is the deviation of the MAR chain from the resonant position in the energy space. The maximum value and the width of the resonance in Eq. (31) is determined by the magnitude of the second term in the denominator, which is of the order of $(n-1)^{2} D^{n}$ at $n>2$ [14]. This results in the peak height $\sim 1 /(n-1)^{2}$ and the width $(n-1) D \delta$, the latter being much smaller than the distance $\delta \sqrt{D}$ between the levels within the cluster. This means that all peaks in the resonant region $\delta \sqrt{D}$ give independent partial contributions into the net current, thus, forming the total contribution of a resonant MAR chain,

$$
J_{n}[\text { chain }] \sim \frac{2 e}{h} n \sum_{j=1}^{n-1} \int_{-\infty}^{\infty} d \varepsilon \frac{D^{2} \delta^{2}}{\varepsilon^{2}+\left[(n-1) a_{j} D \delta\right]^{2}} \sim \frac{2 e}{h} n D \delta \text {. }
$$

The number of such chains can be estimated as $\mathcal{E}_{n}(V) / \delta$, where $\mathcal{E}_{n}(V)$ is the width of the interval of the energy integration in which the resonant trajectories exist (see Fig. 8 where four resonant sets of MAR trajectories are depicted). If the voltage is $V<V_{n-1}$, then this interval is $\mathcal{E}_{n}(V)=n e V-$ $2 \Delta=2 \Delta\left(V / V_{n}-1\right)$ and increases with voltage; at a higher voltage $V>V_{n-1}$, the quantity $\mathcal{E}_{n}(V)=2 \Delta\left(1-V / V_{n-2}\right)$ decreases and turns to zero at $V=V_{n-2}$. Thus, the resonant magnitude of the $n$th current at $e V=k \delta$ is given by

$$
J_{n}^{\mathrm{res}} \sim \frac{2 e}{h} n D \mathcal{E}_{n}(V),
$$

and approaches the maximum value $(2 e / h) 2 n \Delta D /(n-1)$ at $V=V_{n-1}$. The width of the resonance in voltage can be estimated as follows. The deviation in voltage $\Gamma(e V)$ from its resonant value $e V=k \delta$ results in a deviation of the distance $(n-2) \Gamma(e V)$ between the first and the last $n-1$ subgap Andreev reflections. In order to hold the resonant transmissivity, the latter value should not exceed the width of the resonant region $\delta \sqrt{D}$. As the result, the width of the voltage peak at the single-channel IVC [14] is

$$
\Gamma(e V) \sim \delta \sqrt{D} /(n-2) .
$$

In a multichannel junction, the resonant current magnitude in Eq. (33) can be achieved at arbitrary subgap voltage by tuning the level spacing $\delta(\mu)=\mu \delta_{m}$ [a more precise estimate is given by Eq. (13)]. Thus, the main contribution to $J_{n}$ comes from narrow vicinities of the resonant level spacings $\delta_{k}=$ $\mu_{k} \delta_{m}=e V / k$. The width $\Gamma(\mu)$ of the resonant regions of $\mu$ can be estimated similar to the width of the voltage peak in a single-channel case: The deviation in $\delta, \Gamma(\delta)=\Gamma(\mu) \delta_{m}$ times the number of interlevel distances $e V(n-2) / \delta$ between the first and the last subgap Andreev reflections should not exceed $\delta \sqrt{D}$, which yields

$$
\Gamma(\mu) \sim \frac{\delta^{2} \sqrt{D}}{e V(n-2) \delta_{m}},
$$

and therefore the contribution from a given resonant value $\delta_{k}$ to the current is

$$
\begin{aligned}
J_{n}^{(k)} & \sim \mathcal{N} j_{n}^{\text {res }}[\Gamma(\mu) q(\mu)]_{\mu=\mu_{k}} \\
& \sim \frac{2 e}{h} \mathcal{N} \frac{n D^{3 / 2} \mathcal{E}_{n}(V)}{e V(n-2) \delta_{m}}\left(\frac{e V}{k}\right)^{2} q\left(\frac{e V}{k \delta_{m}}\right) .
\end{aligned}
$$

Since $\delta_{k}$ cannot exceed $\delta_{m}$, the contributions to the net current $I_{n}$ come from $I_{n}^{(k)}$ with the numbers $k_{\min }=$ $\operatorname{Int}\left(e V / \delta_{m}\right)+1 \leqslant k<\infty$. Thus, expressing the number of 
channels $\mathcal{N}$ through the normal resistance, we obtain final expression for the $n$-particle current,

$$
I_{n}=C \frac{n \mathcal{E}_{n}(V) \sqrt{D}}{e R_{N}(n-2) \delta_{m}} \sum_{k=k_{\min }}^{\infty} \frac{e V}{k^{2}} q\left(\frac{e V}{k \delta_{m}}\right), \quad n>2,
$$

where $C$ is a numerical constant. The value of this constant $C=0.6$ is found by comparing Eq. (37) with the result of analytical calculation of multiparticle currents by using the phase averaging method in Eq. (28) (see the Appendix).

Such an approach can easily be generalized for the case of the angle-dependent transparency coefficient $D(\cos \theta)$ : The constant factor $\sqrt{D}$ in Eq. (37) must be replaced by the function $\sqrt{D\left(e V / k \delta_{m}\right)}$ within the sum over $k$. For a wide enough barrier, $D(\mu)$ rapidly decreases with $\mu$ and therefore plays the role of a cutoff factor for the contributions of very long trajectories associated with small resonant interlevel distances $\delta_{k}=e V / k \ll \delta_{m}$ at large $k$. As a result, the sharp resonances at $e V=n \delta_{m}$ remain, but the smooth background will undergo noticeable suppression, which brings the IVC closer to the one of the 1D case [14]. Obviously, one should expect a qualitatively similar effect due to decreasing of the electron mean free path or the width $w$ of the junction.

At $e V \gg \delta_{m}\left(k_{\min } \gg 1\right)$, we can approximately replace the summation over $k$ by integration and obtain for both the $2 \mathrm{D}$ and the $3 \mathrm{D}$ cases,

$$
I_{n}(V)=C \frac{n \mathcal{E}_{n}(V) \sqrt{D}}{e R_{N}(n-2)} .
$$

In such an approximation, the partial currents with numbers $n>2$ as functions of voltage have the shape of triangles with the bases between $V_{n}$ and $V_{n-2}$ and with the apex at $V_{n-1}$ having the amplitude $2 n \Delta \sqrt{D} / e R_{N}(n-1)(n-2)$. The net current $I(V)$ at given voltage $V\left(V_{n}<V<V_{n-1}\right)$ contains contributions from two consequent partial currents: The increasing arm of $I_{n}$ and decreasing arm of $I_{n+1}$, therefore, the IVC represents a piecewise linear function connecting apexes of the triangles,

$$
\begin{aligned}
I(V)= & \frac{C \sqrt{D}}{R_{N}(n-2)}\left[(n+2) V-\frac{4 \Delta}{e(n-1)}\right], \\
& V_{n}<V<V_{n-1}, \quad n>2 .
\end{aligned}
$$

Its behavior at the edges of the subgap region is described by the following expressions:

$$
I(V)=C \frac{\sqrt{D}}{R_{N}} \begin{cases}5 V-2 \Delta / e, & e V>2 \Delta / 3, \\ V(1+e V / \Delta), & \delta_{m} \ll e V \ll \Delta .\end{cases}
$$

At lower voltages $e V<\delta_{m}$, Eq. (37) describes powerlike decay of the current with the voltage $I(V) \sim V^{3}$. Equations (39) and (40) as well as the more general Eq. (37) clearly demonstrate the universality of the averaged IVC found by the numerical computation: The smooth part of the dc current at $e V<\Delta$ is the universal function of the applied voltage, and it is independent of the junction length and scales as the $3 / 2$ power of the junction transparency.

The comparison between the results of our numerical calculation and the analytical approach is shown in Fig. 9 where both the general Eq. (37) (the blue solid line) and its averaged form Eq. (39) (the dashed line) were plotted. We see

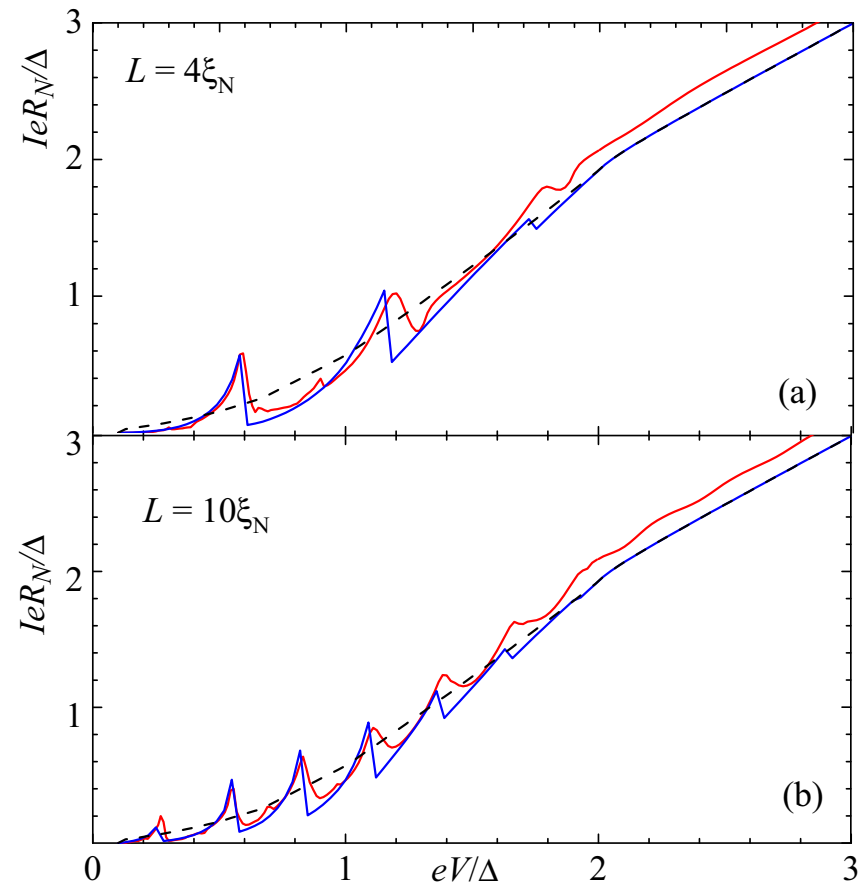

FIG. 9. Analytically calculated IVC [Eq. (37), the blue solid line] and its averaged form [Eqs. (27), (29), and (39), the dashed curve] in comparison with the result of numerical computation (the red solid curve) for a $2 \mathrm{D}$ junction with the transparency $D=0.1$ and lengths (a) $L=4 \xi_{N}$ and (b) $L=10 \xi_{N}$.

that Eq. (37) also describes oscillations of the IVC revealed in numerical computation with sharp peaks at the resonant voltages $e V=k \delta_{m}$ related to the electron trajectories at the reference point $\mu=1$. In the $2 \mathrm{D}$ case, due to divergence of

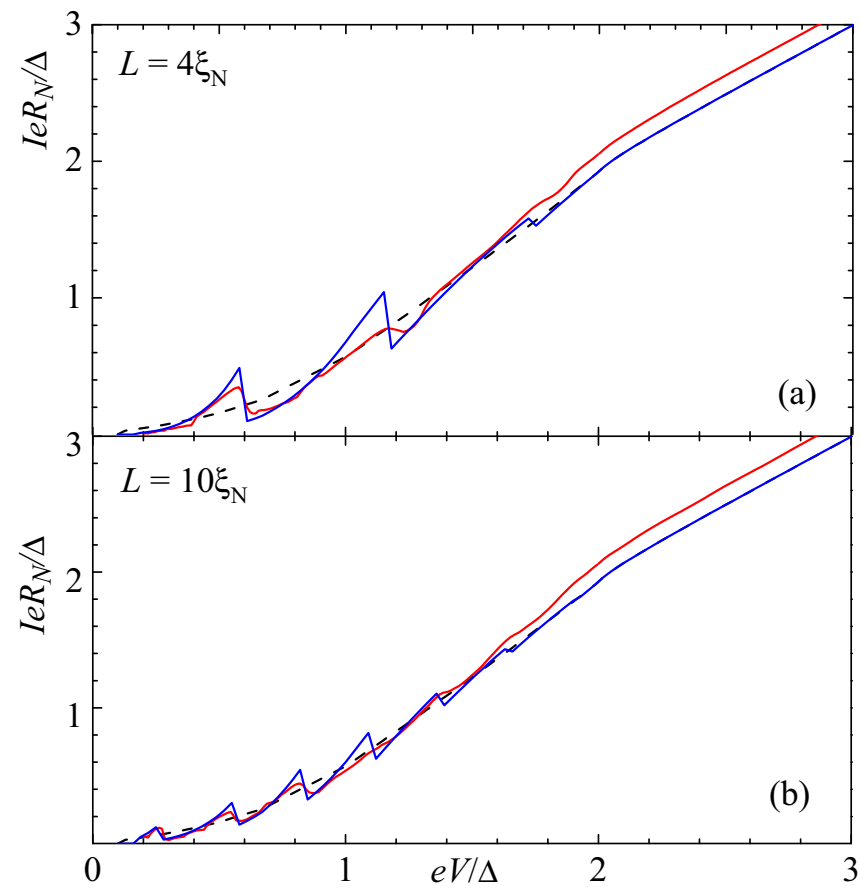

FIG. 10. The same as in Fig. 9 for the 3D junction. 
the geometric weight $q(\mu)$ at the reference point, the formal calculation of IVC by means of Eq. (37) leads to singularities of $I(V)$ at the resonant voltages. A simple regularization of the geometric weight $q(\mu)$ by, e.g., a small shift of the singularity to the region $\mu>1$, eliminates these divergencies. The figure shows satisfactory agreement between the numerical and the analytical results except the absence of the excess current in the approximate expressions Eqs. (27) and (29) for the one- and two-particle currents. A similar conclusion is valid for the 3D junction; in this case shown in Fig. 10, no additional regularization is needed since the geometric weight is nonsingular.

\section{SUMMARY}

In this paper we investigate, both numerically and analytically, the dc current transport at the given applied voltage through the ballistic SNINS junction of finite length $L$ and width. In particular, such a model corresponds to the experimental setup with the normal 2D electron gas confined by massive superconducting electrodes and controlled by a narrow electrostatic gate playing the role of a tunable tunnel barrier of moderately low transparency $D$ (Josephson field effect transistor). Using the coherent multiple Andreev reflections formalism, we found that the characteristic features of the charge transfer in this device are fully determined by the presence of bound Andreev states which provide conditions for the resonant transmissivity.

In the limit of a short junction $L<\xi_{N}$ when only one pair of the levels exists for most of quasiparticles (except those propagating at small angles to the interfaces), the IVC resembles the one of a ballistic point contact with a scatterer: The current decays exponentially with the voltage and exhibits a typical subharmonic structure periodic in $1 / V$. However, in junctions with short but finite lengths, the role of the energy parameter, which defines the period of subharmonics and the decrement of the exponential current decay, is played by the Andreev interlevel distance $\delta_{m}$ rather than the superconducting energy gap in the case of the point contact.

The most interesting phenomena were predicted for long junctions with multiple Andreev levels. The existence of fully resonant MAR trajectories passing through the chain of the levels essentially enhances the subgap current at voltages commensurate with the level spacing thus creating a resonance periodic structure in $V$ with the period $\delta_{m} / e$. Furthermore, the averaged IVC has a power form and exhibits a peculiar universality: It does not depend on the junction length and is universal for all junction transparencies at $e V<\Delta$ being normalized by $\sqrt{D} / R_{N}$. The physical explanation of these characteristic features, discovered in numerics, is given within the framework of the theory of resonant MAR charge transfer. We discuss the effects of the angle dependence of the transmission coefficient and the finite values of the electron-scattering length and the contact width.

Finally, we note that both resonant effects-subharmonics of the interlevel distance in short junctions and its harmonics in long ones-can be used for direct experimental probing of Andreev levels in ballistic SNS structures.

\section{APPENDIX: MULTIPARTICLE CURRENTS}

In this Appendix we present analytical expressions for several multiparticle currents $I_{n}$ calculated in the limits $D \ll 1, L \gg \xi_{N}$ by making use of Eq. (28) taken in the approximation of equidistant Andreev levels. Due to very cumbersome expressions for higher $n$-particle currents, we restrict our examples by $n=3-5$,

$$
\begin{aligned}
I_{3}= & \frac{3 \sqrt{D}}{e R_{N}} \int_{-3 e V / 2}^{E_{\max }} d E \sqrt{N_{0} N_{3}}, \\
E_{\max }= & \min (-\Delta, \Delta-2 e V), \quad 2 / 3<e V / \Delta<2, \\
\phi_{0}= & 2 \gamma_{1}-\gamma_{2}, \quad \phi_{3}=2 \gamma_{2}-\gamma_{1}, \\
I_{4}= & \frac{2 \sqrt{D}}{e R_{N}} \int_{-2 e V}^{E_{\max }} \frac{N_{0} N_{4} d E}{N_{0}+N_{4}}\left[\left(\frac{N_{0}}{N_{4}}\right)^{1 / 4}+\left(\frac{N_{4}}{N_{0}}\right)^{1 / 4}\right] \\
E_{\max }= & \min (-\Delta, \Delta-3 e V), \quad 1 / 2<e V / \Delta<1, \\
\phi_{0}= & (1 / 2)\left(3 \gamma_{1}-\gamma_{3}\right), \quad \phi_{4}=(1 / 2)\left(3 \gamma_{3}-\gamma_{1}\right), \\
I_{5}= & \frac{20 \sqrt{2 D}}{\pi e R_{N}} \int_{-5 e V / 2}^{E_{\max }} d E\left[\int_{0}^{y_{-}}+\int_{y_{+}}^{\infty}\right] d y \\
& \times \frac{\mathrm{A} 2)}{(y-2)^{2}\left[R(y)+8 y^{2}\right] N_{+}^{2}-R(y)(3 y-2)^{2} N_{-}^{2}}, \\
R(y)= & y^{2}-3 y+1, \quad N_{ \pm}=(1 / 2)(3 \pm \sqrt{5}), \\
N_{ \pm}= & N_{0} \pm N_{5}, \quad 2 / 5<e V / \Delta<2 / 3, \\
E_{\max }= & \min (-\Delta, \Delta-4 e V), \quad \phi_{5}=3 \gamma_{3}-2 \gamma_{2} . \\
\phi_{0}= & 3 \gamma_{2}-2 \gamma_{3}, \quad \phi, \quad(y-2) \sqrt{y / R(y)}
\end{aligned}
$$

Here $N_{n}=N\left(E+n e V, \phi_{n}\right)$ is the density of states defined in Eq. (26), and $\gamma_{k}=\arccos [(E+k e V) / \Delta]$.

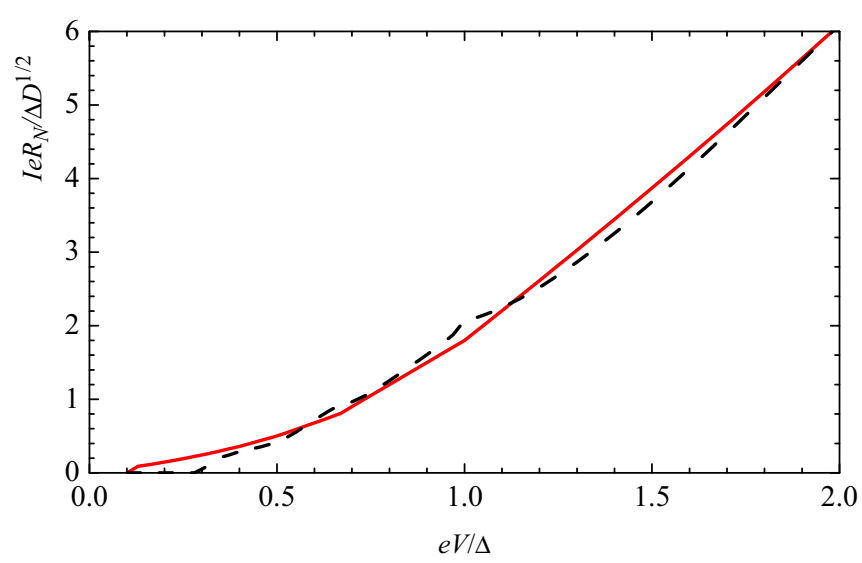

FIG. 11. Subgap IVC calculated from Eq. (39) with the fitting constant $C=0.6$ (the solid line), compared with the sum of seven partial currents evaluated from Eq. (28) by the phase averaging method (the dashed line). 
These equations give the shape of $I(V)$ very close to Eq. (39), which enables us to determine the fitting constant $C=0.6$ in Eqs. (39) and (37). The result of such a fitting is shown in Fig. 11 where the dashed line is the sum of seven partial currents obtained in an analytically tractable form.
[1] H. Takayanagi and T. Kawakami, Phys. Rev. Lett. 54, 2449 (1985).

[2] H. Takayanagi, T. Akazaki, and J. Nitta, Phys. Rev. Lett. 75, 3533 (1995).

[3] T. Schäpers, Superconductor/Semiconductor Junctions (Springer, Berlin, 2001).

[4] A. Chrestin, R. Kürsten, K. Biedermann, T. Matsuyama, and U. Merkt, Superlattices Microstruct. 25, 711 (1999).

[5] T. Akazaki, H. Takayanagi, J. Nitta, and T. Enoki, Appl. Phys. Lett. 68, 418 (1996).

[6] P. Samuelsson, ̊̊. Ingerman, G. Johansson, E. V. Bezuglyi, V. S. Shumeiko, G. Wendin, R. Kürsten, A. Richter, T. Matsuyama, and U. Merkt, Phys. Rev. B 70, 212505 (2004).

[7] T. D. Clark, R. J. Prance, and A. D. C. Grassie, J. Appl. Phys. 51, 2736 (1980).

[8] A. D. Zaikin and G. F. Zharkov, Zh. Eksp. Teor. Fiz. 78, 721 (1978) [Sov. Phys. JETP 51, 364 (1980)].

[9] A. D. Zaikin and G. F. Zharkov, Zh. Eksp. Teor. Fiz. 81, 1781 (1981) [Sov. Phys. JETP 54, 944 (1981)].

[10] A. D. Zaikin and G. F. Zharkov, Pis'ma Zh. Eksp. Teor. Fiz. 35, 465 (1982) [JETP Lett. 35, 636 (1982)].
[11] E. N. Bratus', V. S. Shumeiko, and G. Wendin, Phys. Rev. Lett. 74, 2110 (1995).

[12] D. Averin and A. Bardas, Phys. Rev. Lett. 75, 1831 (1995).

[13] J. C. Cuevas, A. Martín-Rodero, and A. Levy Yeyati, Phys. Rev. B 54, 7366 (1996).

[14] Å. Ingerman, G. Johansson, V. S. Shumeiko, and G. Wendin, Phys. Rev. B 64, 144504 (2001).

[15] E. V. Bezuglyi, A. S. Vasenko, E. N. Bratus', V. S. Shumeiko, and G. Wendin, Phys. Rev. B 73, 220506(R) (2006).

[16] E. V. Bezuglyi, E. N. Bratus', and V. S. Shumeiko, Phys. Rev. B 83, 184517 (2011).

[17] A. V. Zaitsev and D. V. Averin, Phys. Rev. Lett. 80, 3602 (1998).

[18] G. Johansson, G. Wendin, K. N. Bratus', V. S. Shumeiko, Superlattices Microstruct. 25, 905 (1999).

[19] G. de Gennes and D. Saint-James, Phys. Lett. 4, 151 (1963).

[20] B. A. Aminov, A. A. Golubov, and M. Y. Kupriyanov, Phys. Rev. B 53, 365 (1996).

[21] E. V. Bezuglyi, E. N. Bratus', and V. S. Shumeiko, Physica C 499, 15 (2014). 